\title{
Dual perspectives on stigma: reports of experienced and enacted stigma by those affected and unaffected by podoconiosis
}

\author{
Desta Ayode, ${ }^{1}$ Abebayehu Tora, ${ }^{2}$ David Farrell, ${ }^{3}$ Getnet Tadele, ${ }^{1}$ Gail Davey, ${ }^{4}$ \\ Colleen M. McBride ${ }^{5}$ \\ ${ }^{1}$ College of Social Sciences, Addis Ababa University, Addis Ababa, Ethiopia; ${ }^{2}$ Department of \\ Sociology, Wolaita Sodo University, Sodo, Ethiopia; ${ }^{3}$ People Designs Inc., Durham, NC, USA; \\ ${ }^{4}$ Brighton and Sussex Medical School, Falmer, Brighton, UK; ${ }^{5}$ Department of Behavioral Sciences \\ and Health Education, Rollins School of Public Health, Emory University, Atlanta, GA, USA
}

\begin{abstract}
Significance for public health
Disease-related stigma is a public health concern steadily gaining global attention. In this report, we evaluated dual perspectives of stigma, that is, we compared reports of stigma experienced by families affected by a heritable neglected tropical disease with reports of enacted stigma among neighbouring families unaffected by the disease. We found that unaffected neighbours reported enacting less stigma than affected respondents reported experiencing. Levels of enacted and experienced stigma also were influenced by community members' perceptions of the causes of disease. Enacted and experienced stigma levels also varied by gender (males reported less of each) across sites. Taken together, these findings highlight the need for stigma reduction strategies to involve both affected and unaffected groups if interventions are to be effective. These efforts also should address community members' baseline explanations of what causes heritable health conditions while considering gender and community context.
\end{abstract}

\section{Abstract}

Background: Disease-related stigma is a public health concern steadily gaining global attention. Evidence consistently shows that an individual's attribution of disease cause can prompt or justify interpersonal stigma. However, few studies have explored causal beliefs about inherited disease and their influence on stigmatising behaviours in low and middle income countries.

Design and methods: The study was conducted in 2013, in six communities in Wolaita zone, Southern Ethiopia. A total of 1800 respondents took part in the study, 600 were affected by an inherited disease and 1200 were unaffected neighbours. Two versions of the interviewer-administered survey were created, with measures assessed in parallel on experienced stigma for the affected and enacted stigma for unaffected respondents.

Results: Mean levels of enacted stigma reported by unaffected respondents were slightly lower $(2.0, \mathrm{SD}=0.7)$ than experienced stigma reported by affected respondents [2.2 (standard deviation=1.1)]. Beliefs that podoconiosis was hereditary were significantly and positively associated with levels of enacted stigma reported by unaffected respondents and experienced stigma reported by affected respondents $(\mathrm{P}<0.001)$. There was no association of reported levels of stigma experienced by affected respondents with levels of enacted stigma reported by the neighbouring unaffected respondents. Males consistently reported significantly lower levels of experienced and enacted stigma than females, $\mathrm{P}<0.0001$.
Conclusions: If stigma reduction interventions are to be successful, culturally tailored, gender inclusive and innovative health education programs are required, directed at the general community as well as individuals affected by inherited diseases.

\section{Introduction}

Over the past several decades, disease-related stigma has gained international attention as a serious public health concern. ${ }^{1}$ A broad array of research has emerged showing that stigma associated with Neglected Tropical Diseases (NTDs) contributes substantially to the disease burden of affected communities. ${ }^{2}$ Available evidence indicates that those affected by NTDs report a host of negative experiences including social isolation, and discrimination. In turn, these experiences can decrease the effectiveness of disease prevention and control through their negative influence on treatment-seeking and adherence to preventive recommendations. ${ }^{3-5}$ Experiences of stigma also negatively influence psychosocial well-being of affected individuals. Individuals with stigmatised disease may feel fear or shame that can lead to anxiety and depression. This in turn may lead to decreased social participation and social exclusion. ${ }^{6}$ Indeed, several stigma-related reports have suggested that the consequences of stigma outweigh the burden of the underlying physical affliction. ${ }^{7}$

Stigma is interpersonally experienced, comprising a number of cooccurring behaviours that disrupt social relationships and access to resources. ${ }^{1}$ Scambler's hidden distress model $^{8}$ suggests a dual process in which those unaffected by a condition enact stigmatising behaviours such as using their power to discriminate unfairly, ostracise or actively trouble others whom they regard to be unacceptable. In turn, those targeted by stigma, experience loss of status, discrimination and exclusion.

Evidence also shows that levels of stigma are influenced by what community members perceive to be the causes of disease, henceforth referred to as causal attributions. ${ }^{2}$ For example, perceiving that health conditions run in families has been linked to stigma. ${ }^{9}$ This can arise from perceptions that carrying a gene mutation labels a group as fundamentally different and even defective in their genetic makeup. ${ }^{10}$ Accordingly, in the developed world, studies of mental illness and other conditions have demonstrated that increased stigma is associated with attributions that a disease is hereditary. ${ }^{11,12}$ However, very little of this research has been conducted in low and middle income countries. The limited available evidence, however, supports similar associations of genetic causal attributions for diseases with increased stigma. ${ }^{13}$ 
Additionally, the majority of research on stigma in the context of inherited diseases has focused on the perspective of affected groups without evaluating in parallel the perceptions of the broader community. Doing so within a shared social environment such as a community could point to traction points for genetics education interventions aimed to reduce stigma.

The occurrence of stigma has been well-documented in the context of podoconiosis patients. ${ }^{14}$ Podoconiosis (endemic non-filarial elephantiasis) is a non-infectious disease caused by exposure of bare feet to volcanic red clay soil. The disease mostly affects subsistence farmers who work barefoot, particularly on red clay soils of volcanic areas. ${ }^{15,16}$ Podoconiosis is a chronic, debilitating disorder and a considerable public health problem in Asia, Central America and tropical Africa, including Ethiopia. ${ }^{17}$ Recent nationwide mapping suggests overall disease prevalence of $4 \%{ }^{18}$ and about 1 in 20 people are estimated to be affected by the condition in Wolaita Zone of southern Ethiopia. ${ }^{19,20}$ Evidence suggests that both genetics and environmental factors are involved in the pathogenesis of podoconiosis and that heightened susceptibility to soil exposure clusters in families; the estimated heritability is $63 \%{ }^{21}$ The disease is entirely preventable if genetically high-risk individuals consistently protect their feet by wearing shoes and practicing foot hygiene. ${ }^{15,16,22}$

The onset of podoconiosis causes a progressive bilateral swelling of the lower legs. Patients with the condition can be identified readily. Prior studies have documented that these individuals experience stigma related to physical disfigurement and emitting an offensive odour as the disease advances and to inability to work or meet family responsibilities. ${ }^{9,23}$ Together, these place considerable psychosocial burdens on affected individuals. Social stigmatisation of people with the disease is widespread and patients are often excluded from social activities and interactions and are not allowed to marry into unaffected families (Figure 1). ${ }^{24-26}$

Misconceptions that fuel stigma and discrimination include notions that the disease cannot be prevented, treated or controlled and that it runs in families through inevitable hereditary factors. ${ }^{23,25}$ Studies in southern Ethiopia have showed that $93 \%$ of respondents held at least one misconception about the cause of podoconiosis and more than half (56\%) showed stigmatising attitudes towards social interactions with affected individuals. ${ }^{25}$

In our previous qualitative research, we found that beliefs that podoconiosis was caused by heredity were acknowledged both by affected and unaffected individuals and that these beliefs influenced partner selection and marital stability. ${ }^{14}$ Affected adults talked about the effect of their disease on their children's social interactions and its negative impact on social opportunities, such as attending school. However, our earlier work had several limitations. Results were largely based on group interviews in which men were more vocal participants. As with previous studies of stigma, the majority of participants in the group interviews were affected respondents who volunteered to participate. Thus, we gained little insight into unaffected individuals' perceptions of their own stigmatising behaviours in association with causal beliefs. Moreover, we were not able to assess whether the stigma experiences of affected individuals aligned with unaffected individuals' reports of stigmatising behaviours. Additionally, in the Wolaita area, a non-governmental organisation (NGO), Mossy Foot International (MFI), has been providing educational and clinical services to a growing number of affected families over the last decade. Thus it is possible that causal beliefs and stigma experiences could differ by duration of relationship of the NGO in these communities. Lastly, our qualitative approaches did not enable us to assess potential gender difference regarding the extent to which stigma may affect men and women differently with respect to marital relations and other social interaction behaviours.

The purpose of this report is therefore, to quantitatively evaluate, in a large community-based sample, dual perspectives regarding stigma.
We compared reports of stigma experienced by respondents from families with podoconiosis (henceforth referred to as affected) with reports of stigma enacted by respondents without the condition (henceforth referred to as unaffected). We posed three primary research questions: i) what are the levels of enacted and experienced stigma? Do these levels differ by gender or across communities? ii) Are beliefs about the cause of podoconiosis associated with the levels of enacted and experienced stigma? iii) Are the levels of experienced stigma reported by affected respondents associated with levels of enacted stigma reported by unaffected neighbour respondents?

\section{Design and methods}

\section{Study setting and participants}

The study was conducted in the Wolaita zone of Southern Ethiopia, an area endemic for podoconiosis. The prevalence of podoconiosis is $5.5 \%{ }^{19}$ The total population of the zone is estimated to be 1.7 million with $88 \%$ living in rural settings. Residents are largely of Wolaita ethnicity, a homogenous cultural and religious (Christian) background. ${ }^{27}$ Most people earn their living from subsistence farming. The zone is divided into 15 administrative districts or communities. Six communities that were receiving podoconiosis prevention and treatment services from a local NGO (described below) were selected to participate. Data presented are taken from the baseline survey of an intervention trial described elsewhere. ${ }^{28}$ Briefly, the selected communities were those serving the largest number of affected families to enable rapid recruitment. A total of 1800 participants took part in the on-going intervention trial, 300 in each site, of which 100 were from affected families and 200 from unaffected families. The shoe distribution ledgers of the NGO, Mossy Foot International (MFI), were used as the sampling frame to identify a random sample of children ages 3 to 6 years from affected households. Where more than one age-eligible child (i.e., ages 3 to 6 ) was listed, the child with the next birthday was selected as the index child for the parent to consider when answering survey questions. Two neighbouring unaffected households were then selected for each affected family using the following criteria: i) no one in the household was a blood relative of anyone living in the matched affected household; ii) neither parent in the household had a first degree relative with podoconiosis; iii) the household included at least one child between

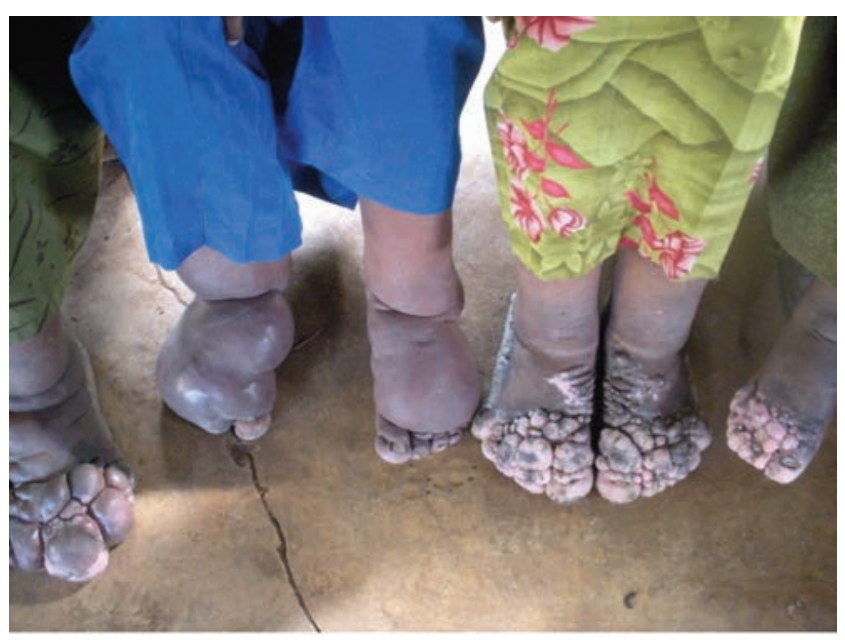

Figure 1. Bilateral swelling of the lower legs caused by podoconiosis (Photo by J. Shortall). 
the ages of 3-6; iv) the household was within 500 meters of the affected household; and v) an adult in the household agreed to participate.

\section{Ethics statements}

Ethical approval was obtained for all study procedures from the National Ethical Review Committee of the Ethiopian Science and Technology Ministry and the Institutional Review Board of the National Human Genome Research Institute. A written informed consent was obtained from all participants. All the data were anonymous and personal identifications of the respondents were coded and stored separately. Thumbprints were taken from those who were not able to sign on the information sheet and consent forms.

\section{Survey interviews}

Two versions of the baseline survey were developed that included parallel questions for participants in the affected and unaffected households. Two categories of beliefs were assessed: endorsement that podoconiosis is caused by heredity, or by contagion. Three statements were posed about heredity (e.g., If there's podoconiosis in the family there is nothing that can be done to prevent the disease, agreement with each statement was summed and ranged from 0 to 3 ). Two statements assessed belief about contagion as a cause of podoconiosis (e.g., Podo is contagious, summed agreement ranged from 0 to 2 ). Additionally, we coded each of the five statements as true (1) or false (0) to compute accuracy of understanding which ranged from 0 to 5 .

\section{Experienced stigma (affected households)}

Participants were asked to rate the frequency (never, once or twice, several times, most of the time) of negative interpersonal experiences (e.g., people cut down visiting you) that had occurred in the prior three months. Items for each domain were summed and averaged to arrive at scores ranging from one to four, where higher scores were indicative of more experienced stigma (Internal reliability $=0.94$ ).

\section{Enacted stigma (unaffected households)}

A measure of preferred social distance was adapted for the context of podoconiosis. Participants were presented with a hypothetical situation regarding Mrs Alebo who has started to show signs of foot swelling and is unable to walk. Respondents were asked their willingness to be in six levels of proximity to Mrs Alebo (e.g., to live next door, share meals, work with her on household chores; range = 1-definitely yes, 2maybe, 3-definitely no). Mean scores were calculated from responses to these six items, where higher scores show higher enacted stigma (Cronbach's alpha $=0.91$ ).

All the instruments were translated into the local language (Wolytigna), pilot tested and administered by trained data collectors.

\section{Data collection}

Trained research assistants visited the index children's households and obtained consent from the adult care-giver to complete the survey (described in more detail in McBride and colleagues, 2015). ${ }^{28}$ The adult identified as spending the most time with the [index child] and knowing the child's daily habits the best was asked to complete the baseline survey (survey questions were read to the participant). The baseline data collection was carried out for 15 days in each site in February 2013.

\section{Data analysis}

Data entry and cleaning was done by qualified consultants using EPI Info software (Center for Surveillance, Epidemiology \& Laboratory Services, Atlanta, GA, USA), and then reviewed and crosschecked by data analysts. Analysis of variance was used to test whether mean levels of stigma varied by site. Mean differences in stigma (enacted or experienced) by gender were estimated in a regression model that included terms for the six sites. Relationships between stigma (enacted or endorsed) and beliefs about causes (heredity, contagion, or both) were estimated in linear regression models that controlled for site and gender. To assess whether stigma experienced by affected households was related to the stigma enacted by their neighbouring households, a single measure representing the sum of the enacted sigma scores of the two matched unaffected household was attached to the record for the corresponding affected household. A linear regression model was fitted to the data, where experienced stigma was the dependent meas ure and the sum score of the two unaffected households entered the model as an independent variable. The models also included terms to control for site and gender (of the respondent in the affected household). Additional models included interaction terms to test for variation among sites in the relationship of enacted to experienced stigma.

\section{Results}

\section{Sample characteristics}

Of the 1800 participants (600 from affected households and 1200 from unaffected) approached, 1784 [596 affected (99.3\%) and 1188 unaffected (99\%)] completed the baseline survey (giving an overall response rate of $99.1 \%$ ). As shown in Table 1, the majority of caregivers were females. Affected respondents were significantly older than unaffected respondents (33.9, range $=18-70, \mathrm{SD}=8.7)$ and 31.3 [range $=18-80, \mathrm{SD}=7.4$, respectively, $(\mathrm{P}<0.001)$ ] and significantly less likely to have attended formal schooling than unaffected respondents (43.3 vs. 54.1\%, respectively, $\mathrm{P}<0.001$ ). Affected respondents reported significantly larger household sizes than unaffected respondents (6.4 vs. 5.8, respectively, $\mathrm{P}<0.001)$.

\section{Levels of experienced and enacted stigma}

As shown in Table 2, mean levels of experienced stigma reported by affected respondents was 2.2 ( $\mathrm{SD}=1.1$, 1-low to 3 high). Levels of enacted stigma (1-low to 3 - high) reported by the two unaffected neighbouring households of each affected participant were summed and averaged. The overall mean for levels of reported enacted stigma was slightly lower $(\mathrm{M}=2.0, \mathrm{SD}=0.7)$.

Reported levels of experienced and enacted stigma differed significantly across the six study sites $(\mathrm{P}<0001)$. Affected respondents' reports of experienced stigma scores ranged from 1.2 to 3.5 across communities, while unaffected respondents' reports of enacted stigma ranged from 1.5 to 2.4. There also were significant differences by gender in reports of experienced and enacted stigma. Males consistently reported significantly lower levels of experienced and enacted stigma than females $(\mathrm{P}<0.0001)$.

Levels of experienced stigma reported by females ranged from 1.2 to 3.5 across communities. For males, mean levels of experienced stigma were slightly lower ranging from 0 to 3.2 . Similarly, mean levels of enacted stigma for females ranged from 1.5 to 2.4 , and were slightly higher compared to males, ranging from 1.4 to 2.3. The duration of the relationship between communities and the NGO ranged from 7 tol1 years; there was no uniform alignment between stigma levels and length of this relationship.

\section{Association of beliefs about causes of podoconiosis and stigma level}

Respondents' beliefs that podoconiosis was hereditary were significantly and positively associated with reported levels of experienced stigma among affected respondents and enacted stigma for unaffected respondents (Table 3). Similarly, respondents' beliefs that contagion 
caused podoconiosis were also significantly and positively associated with reports of experienced stigma for affected respondents, and enacted stigma for unaffected respondents. The coefficient values in both regression models indicated that for every unit increase in belief about heredity or contagion as a cause of podoconiosis among affected respondents, experienced stigma increased by an average of 11 and $1 \%$, respectively. Similarly for every unit of increase in belief about heredity or contagion as a cause of podoconiosis among unaffected respondents, enacted stigma increased by an average of 10 and $16 \%$, respectively.

Given the sizable differences in reports of experienced and enacted stigma, we adjusted the prior analyses for site and gender. For affected respondents, only the association of believing that heredity was a cause of podoconiosis remained significantly associated with levels of experienced stigma. For unaffected respondents, beliefs in heredity or contagion were significantly associated with enacted stigma $(\mathrm{P}=0.014$ and $\mathrm{P}=0.042$, respectively).

\section{Association of experienced and enacted stigma}

To test the association of experienced stigma with reports of enacted stigma between the affected and the two matched neighbour households, we first considered the mean levels of enacted stigma for the two neighbours (enacted-sum). There was no association between levels of stigma experienced by affected households with corresponding levels of enacted stigma reported by the neighbouring unaffected households. Further exploration showed that the correlations between the two matched neighbours reports of enacted stigma were quite low $(r=0.10)$. Thus, we tested the association of the highest value of enacted stigma reported by the two neighbours (enacted-high) with levels of experienced stigma among the corresponding affected household. Again, there was no association of experienced or enacted stigma. We also confined the analyses to just affected households that experienced any stigma and again found no association.

\section{Discussion}

These results showed that those affected by podoconiosis consistently reported experiencing more stigma than their unaffected neighbours reported enacting. These findings are consistent with stigma related to podoconiosis found in other regions of Ethiopia. ${ }^{26}$ By contrast, dual perspectives on stigma related to other tropical disease showed lower reports of experienced stigma among those affected by Buruli Ulcer in Ghana compared to levels of enacted stigma reported by those unaffected by the condition. ${ }^{29}$ Given the scarcity of similar studies considering dual perspectives, further research is needed to confirm these results.

We also found significant variation of reported experienced and enacted stigma scores across communities despite their being relatively homogeneous in socio-cultural context. We considered whether this variation might have been due to differences in the NGO's delivery of educational content. Like many NGOs, MFI relies on lay health workers, largely recovered patients, to deliver interventions that may have contributed to differences. However, there was no observable pattern in the

Table 1. Descriptive characteristics of respondents.

\begin{tabular}{|c|c|c|c|}
\hline \multirow[t]{2}{*}{ Characteristics } & \multicolumn{3}{|c|}{ Unaffected $(\mathrm{N}=1188)$} \\
\hline & Affected $(\mathrm{N}=596)$ & & P-values \\
\hline Mean age (SD) & $31.3(7.4)$ & $33.9(8.7)$ & $<0.001$ \\
\hline Respondent male, $\%$ & 13 & 15 & 0.149 \\
\hline Respondent ever gone to school, $\%$ & 54.1 & 43.3 & $<0.001$ \\
\hline Mean household size & $5.8(1.7)$ & $6.4(1.9)$ & $<0.001$ \\
\hline Mean enacted stigma (scale: $\min 2 \max 6$ ) & $2.0(0.7)$ & - & - \\
\hline Mean experienced stigma (scale: $\min 1 \max 4$ ) & - & $2.2(1.1)$ & - \\
\hline
\end{tabular}

SD, standard deviation

Table 2. Duration of community's relationship with the nongovernmental organisation (NGO) by mean levels of experienced and enacted stigma and respondent gender.

\begin{tabular}{lccccc} 
Community & $\begin{array}{c}\text { Relationship length } \\
\text { with NGO, years }\end{array}$ & $\begin{array}{c}\text { Affected reports: experienced stigma } \\
\text { Female }\end{array}$ & $\begin{array}{c}\text { Unaffected reports: enacted stigma } \\
\text { Female }\end{array}$ & $\begin{array}{c}\text { Male } \\
5\end{array}$ \\
\hline 1 & 3.2 & 3.2 & 1.5 & 1.4 \\
1 & 10 & 1.2 & 1.1 & 2.0 & 2.0 \\
\hline 3 & 08 & 3.5 & 3.1 & 2.4 & 2.3 \\
4 & 08 & 1.8 & 2.0 & 1.9 & 1.8 \\
\hline 6 & 08 & 1.3 & 1.2 & 2.1 & 2.0 \\
2 & 07 & 2.3 & $*$ & 2.4 & 2.1 \\
\hline *Nomen surveyed & & & & &
\end{tabular}

Table 3. Relations between stigma and causal beliefs about podoconiosis.

\begin{tabular}{lcccc} 
Beliefs about causes of podoconiosis & \multicolumn{2}{c}{$\begin{array}{c}\text { Enacted Stigma } \\
\text { (unaffected=1188) }\end{array}$} & & $\begin{array}{c}\text { Experienced stigma } \\
\text { (affected=596) }\end{array}$ \\
& Beta value & P-value & Beta value & 0.11 \\
Model 1: Heredity & 0.10 & 0.047 & 0.0001 \\
Model 2: Contagion & 0.16 & 0.006 & 0.01 & 0.031 \\
\hline
\end{tabular}


differences that aligned with the duration of the community's relationship with the NGO. It is also possible that differences in the pace of affected individuals' recovery from the condition after starting treatment influenced these differences. Our previously described focus group discussions with respondents in communities suggested that respondents who had seen more patients cured in their neighbourhoods reported less fear in terms of social interactions with patients. ${ }^{14}$ Taken together, these differences suggest that pre-planning would be advised to include community-specific needs assessments to evaluate baseline levels and sources of stigma prior to intervention development. Gender also played a role in differences in reports of stigma. Male respondents reported consistently less experienced and less enacted stigma than females. This result is consistent with recent research on Onchocercial disease in five African settings: Cameroon, Ghana, Nigeria (Awka and Ibadan) and Uganda. ${ }^{30}$ This qualitative study revealed that stigma was experienced differently by men and women. Likewise, TB-related studies in Bangladesh and India ${ }^{31}$ have identified significantly higher stigma indices among women than men; as did a study of leprosy-related stigma in India. ${ }^{32}$ This may be explained by women being more disadvantaged by overt disfiguring disease than men because of their greater dependence on physical appearance for social acceptance, marriage and well-being. ${ }^{30}$ It is possible that social consequences such as inability to find a marital partner or the possibility of divorce makes stigma worse for females with podoconiosis than males. Contrary to this, recent studies related to NTDs such as Buruli Ulcer and podoconiosis found no significant difference between the stigma scores of men and women. ${ }^{24,29}$ Thus, the role of gender in stigma is likely to be affected by community context as well and also should be considered as part of intervention planning.

Levels of experienced and enacted stigma were also influenced by respondents' beliefs about the causes of podoconiosis. Beliefs that the condition was heredity or contagious were positively associated with reports of both experienced and enacted stigma. These findings are consistent with prior research in the sphere of mental illness, particularly schizophrenia, showing that beliefs that these conditions are hereditary were associated with desire for greater social distance from those affected. ${ }^{11,12,33}$ Additionally, a study in Zambia found that people with epilepsy who believed their condition to be contagious, or thought their community believed epilepsy to be contagious reported more felt stigma. $^{7,34}$ Thus, an important future direction is to consider community members' explanations of what causes health conditions in designing public health interventions. Identifying gaps in understanding of disease causes using a mental models approach could provide educational and skills building targets for interventions. ${ }^{35}$

Lastly, we found no significant associations between experienced stigma and reports of enacted stigma by neighbours, regardless of how we quantified enacted stigma. To our knowledge, there has been no prior quantitative research that has explored this dual perspective in the context of inherited disease. Thus our interpretation of this null finding must be tentative. In this study we purposely selected neighbours, who by definition were in relatively close proximity to the affected households. Thus our sample may not reflect community-wide levels of enacted stigma. Other studies of the influence of proximity to patients on stigma response in low income communities in Nepal, ${ }^{36}$ showed that people living at a greater distance had the highest perceived stigma score compared to those living closer. Given the strong socio-cultural ties and communal lifestyle of the rural community, the influence of social desirability also could lead neighbours to downplay enacted stigma because they don't want to be perceived negatively. It could also be that even low levels of enacted stigma were perceived so negatively by affected individuals that it inflated their reports.

The large sample size and use of affected-unaffected clusters represent important strengths of this study. There are however some limitations, which may affect generalisation to other cultural settings. The baseline survey was not accompanied by qualitative research that would enable interpretation of, for example, the variation in experienced and enacted stigma between sites. Our partnership with MFI in conducting the study (using their infrastructures and target project sites) may have resulted in some social desirability bias. Even given this, we believe that the findings of the current study have implications for future research and prevention interventions in podoconiosis endemic communities.

\section{Conclusions}

In this southern Ethiopian population, we demonstrated that levels of experienced and enacted stigma were associated with beliefs about the cause of podoconiosis. Misconceptions about the causes of podoconiosis exacerbated stigmatising attitudes and practices. Current interventions to reduce stigma in these communities are targeted chiefly to affected families; extension to the rest of the community is limited. If stigma reduction interventions are to be successful, culturally-tailored, gender inclusive and innovative health education programs directed at the broader community will be needed. There is considerable conceptual support for educating lay audiences about the role of genes and environment in disease causation as part of comprehensive health promotion interventions. ${ }^{14,37}$ For instance, community health education programs focused on reducing stigma and increasing acceptance of patients have been proven to be effective in promoting more favourable attitudes in leprosy and neglected tropical diseases. ${ }^{38,39}$ Secondly and perhaps more importantly, it is necessary to understand that in Ethiopia, like in many sub-Saharan African rural communities, close family ties, communal living situations and traditional belief systems undoubtedly influence the expressions for stigmatisation.

Correspondence: Colleen M. McBride, Department of Behavioral Sciences and Health Education, Rollins School of Public Health, Emory University, 1518 Clifton Road NE, GCR 564, Atlanta, GA 30322, USA.

E-mail: cmmcbri@emory.edu

Key words: Enacted stigma; Experienced stigma; Podoconiosis.

Acknowledgements: we would like to thank Mossy Foot International (MFI) in Southern Ethiopia and all participants of the study, Ejigayehu Global Trading and Development Plc, for data encoding and Christopher Price, for analysing the data.

Contributions: CM and GD participated as principal investigators, oversaw study design, led the study process analysed and interpreted results, and manuscript preparation. DA developed survey tools, oversaw data-collection, interpreted data analyses and led manuscript preparation. AT participated in development of survey tools, fielding data collection, assisted in manuscript preparation. GT assisted in interpretation of findings and manuscript preparation. DF designed and pilot tested educational materials, assisted in interpretation of results and manuscript preparation.

Conflict of interest: the authors declare no potential conflict of interest. Funding: funding was provided by the Intramural Research Program of the National Human Genome Research Institute.

Received for publication: 6 February 2016.

Accepted for publication: 6 May 2016.

(C) Copyright D. Ayode et al., 2016

Licensee PAGEPress, Italy

Journal of Public Health Research 2016;5:689

doi:10.4081/jphr.2016.689

This work is licensed under a Creative Commons Attribution NonCommercial 4.0 License (CC BY-NC 4.0). 


\section{References}

1. Hatzenbuehler ML, Phelan JC, Link BG. Stigma as a fundamental cause of population health inequalities. Am J Pub Health 2013;103:813-21.

2. Weiss M. Stigma and the social burden of neglected tropical diseases. PLoS Neglect Trop D 2008;2:e237.

3. Ribera J, Grietens K, Toomer E, Hausmann-Muela S. A word of caution against the stigma trend in neglected tropical disease research and control. PLOS Neglect Trop D 2009;3:e445.

4. Tora A, Davey G, Tadele G. Factors related to discontinued clinic attendance by patients with podoconiosis in southern Ethiopia: a qualitative study. BMC Pub Health 2012;12:e902.

5. Perera M, Whitehead M, Molyneux D, et al. Neglected patients with a neglected disease? A qualitative study of lymphatic filariasis. PLoS Neglect Trop D 2007;1:e128.

6. Adhikari B, Kaehler N, Chapman R, et al. Factors affecting perceived stigma in leprosy affected persons in western Nepal. PLOS Neglect Trop D 2014;8:e2940.

7. Van Brakel W. Measuring leprosy stigma - a preliminary review of the leprosy literature. Int J Lepr Other Mycobact Dis 2003;71:19087.

8. Scambler G. Stigma and disease: changing paradigms. Lancet 1998;352:1054-5.

9. Tekola F, Bull S, Farsides B, et al. Impact of social stigma on the process of obtaining informed consent for genetic research on podoconiosis: a qualitative study. BMC Med Ethics 2009;10:1-10.

10. WonPat-Borja A, Yang L, Link B, Phelan J. Eugenics, genetics, and mental illness stigma in Chinese Americans. Soc Psychiatry Psychiatr Epidemiol 2012;47:145-56.

11. Jorm AF, Griffiths KM. The public's stigmatising attitudes towards people with mental disorders: how important are biomedical conceptualisations? Acta Psychiatr Scand 2008;118:315-21.

12. Dietrich S, Beck M, Bujantugs B, et al. The relationship between public causal beliefs and social distance toward mentally ill people. Aust N Z J Psychiatry 2004;38:348-54.

13. Chapple A, May C, Campion P. Lay understanding of genetic disease: A British study of families attending a genetic counseling service. J Genet Counsel 1995;4:281-300.

14. Ayode D, McBride M, de Heer H, et al. The association of beliefs about heredity with preventive and interpersonal behaviors in communities affected by podoconiosis in rural Ethiopia. Am J Trop Med Hyg 2012;87:623-30.

15. Davey G, GebreHanna E, Adeyemo A, et al. Podoconiosis: a tropical model for gene-environment interactions? Trans R Soc Trop Med Hyg 2007;101:91-6.

16. Molla Y, Le Blond J, Wardrop N, et al. Individual correlates of podoconiosis in areas of varying endemicity: a case-control study. PLOS Neglect Trop D 2013;7:e2554.

17. Korevaar D, Visser B. Podoconiosis, a neglected tropical disease. Neth J Med. 2012;70:210-4.

18. Deribe K, Brooker SJ, Pullan RL, et al. Epidemiology and individual, household and geographical risk factors of podoconiosis in Ethiopia: results from the first nationwide mapping. Am J Trop Med Hyg 2015;92:148-58.
19. Destas K, Ashine M, Davey G. Prevalence of podoconiosis (endemic non-filarial elephantiasis) in Wolaitta, Southern Ethiopia. Trop Doct 2003;33:217-20.

20. Davey G. Podoconiosis, non-filarial elephanthiasis and lymphology. UAiR Lymphol 2010;43:168-77.

21. Tekola Ayele F, Adeyemo A, Finan C, et al. HLA Class II locus and susceptibility to podoconiosis. N Engl J Med 2012;366:1200-8.

22. Alemu G, Ayele F, Daniel T, et al. Burden of podoconiosis in poor rural communities in gulliso woreda, West Ethiopia. PLOS Neglect Trop D 2011;5:e1184.

23. Tora A, Davey G, Tadele G. A qualitative study on stigma and coping strategies of patients with podoconiosis in Wolaita zone, Southern Ethiopia. Int Health 2011;3:176-81.

24. Tora A, Franklin H, Deribe K, et al. Extent of podoconiosis-related stigma in Wolaita Zone, Southern Ethiopia: a cross-sectional study. Springer Plus 2014;3:647.

25. Yakob B, Deribe K, Davey G. High levels of misconceptions and stigma in a community highly endemic for podoconiosis in southern Ethiopa. Trans R Soc Trop Med Hyg 2008;102:439-44.

26. Deribe K, Tomczyk S, Mousley E, et al. Stigma towards a neglected tropical disease: felt and enacted stigma scores among podoconiosis patients in Northern Ethiopia. BMC Pub Health 2013;13:1178.

27. CSA. The 2007 population and housing census of Ethiopia. Addis Ababa: CSA; 2007.

28. McBride CM, Price CS, Ayode D, et al. A cluster randomized intervention trial to promote shoe use by children at high risk for podoconiosis. IJHSR 2015;5:518-28.

29. Stienstra Y, Van der Graaf W, Asamoa K, Van der Werf T. Beliefs and attitudes toward buruli ulcer in Ghana. Am J Trop Med Hyg 2002;67:207-13.

30. Vlasso C, Weissb M, Ovugac E, et al. Gender and the stigma of onchocercal skin disease in Africa. Soc Sci Med 2000;50:1353-68.

31. Somma D, Thomas B, Karim F, et al. Gender and socio-cultural determinants of TB-related stigma in Bangladesh, India, Malawi and Cambodia. Int J Tuberc Lung Dis 2008;12:856-66.

32. Rao S, Garole V, Walawalkar S, et al. Gender differentials in the social impact of leprosy. Lepr Rev 1996;67:190-9.

33. Ertugrul A, Ulu B. Perception of stigma among patients with schizophrenia. Soc Psychiatry Psychiatr Epidemiol 2004;39:73-7.

34. Atadzhanov M, Haworth A, Chomba EN, et al. Epilepsy-associated stigma in Zambia: what factors predict greater felt stigma in a highly stigmatised population? Epilepsy Behav 2010;19:414-8.

35. Austin LC, Fischhoff B. Injury prevention and risk communication: a mental models approach. Inj Prev 2012;18:124-9.

36. Adhikari B, Shrestha K, Kaehler N, et al. Community attitudes towards leprosy affected persons in Pokhara municipality of western Nepal. J Nepal Health Res Counc 2013;11:264-8.

37. Leventhal $\mathrm{H}$, Breland J, Mora P, Leventhal E. Lay representations of illness and treatment: a framework for action. In: Steptoe A, ed. Handbook of Behavioral Medicine. Springer: New York; 2010. pp 137-54.

38. Raju M, Rao P, Mutatkar R. A study on community-based approaches to reduce leprosy stigma in India. Indian J Lepr 2008;80:267-73.

39. Alonso LM, Alvar J. Stigmatizing neglected tropical diseases:a systematic review. Soc Med 2010;5:218-27. 